Cita bibliográfica: Sánchez-Rivas García, J., Pérez-Hidalgo, M. D., Pablo-Romero Gil-Delgado, M. P. y CamúñezRuiz, J. A. (2019). La demanda del turismo europeo sobre Andalucía medida a través de los alojamientos hoteleros en 2017: un modelo de gravedad. Investigaciones Turísticas (18), pp. 119-137. http://dx.doi.org/10.14198/ INTURI2019.18.06

\title{
La demanda del turismo europeo sobre Andalucía medida a través de los alojamientos hoteleros en 2017: un modelo de gravedad
}

European tourism demand for Andalucía measured through hotel accommodation in 2017: a gravity model

Javier Sánchez-Rivas García iD, Universidad de Sevilla, España sanchezrivas@us.es

María Dolores Pérez-Hidalgo (iD, Universidad de Sevilla, España mdperez@us.es

María del Pópulo Pablo-Romero Gil-Delgado iD, Universidad de Sevilla, España mpablorom@us.es

José Antonio Camúñez-Ruiz iD, Universidad de Sevilla, España camunez@us.es

\section{RESUMEN}

La demanda turística sobre Andalucía (región al sur de España), desde los países europeos, es medida a través del número de turistas alojados en establecimientos hoteleros, para el año 2017. Un modelo econométrico de gravedad sencillo explica dicha demanda, usando elasticidades, a través del PIB de cada país y de la distancia en kilómetros desde dicho país a Andalucía. La obtención de pendiente cercana a la unidad con respecto al PIB nos permite definir una nueva variable, la demanda relativizada por el PIB, y modelizar la misma a partir de la distancia. La pendiente negativa de esta nueva modelización permite plantear la formulación de políticas que minimicen el factor distancia de los países europeos a Andalucía si se quiere incrementar la demanda.

Palabras clave: Andalucía, demanda turística, modelos de gravedad, PIB, distancia en kilómetros.

\section{ABSTRACT}

Tourism demand for Andalusia (southern region of Spain), from European countries, is measured through the number of tourists staying in hotel establishments, during the year 2017. A simple econometric model explains this demand, using elasticities, through the GDP of each 
country and the distance in kilometers from that country to Andalusia. Obtaining a slope close to the unit with respect to GDP allows us to define a new variable, the demand relativised by GDP, and to model it based on distance. The negative slope of this new modeling allows the formulation of policies that bring the European countries closer to Andalusia if demand is to be increased.

Keywords: Andalusia, tourism demand, gravity models, GDP, distance in kilometers.

\section{INTRODUCCIÓN}

Los modelos de gravedad son ecuaciones de relación entre variables, que suelen usarse bajo enfoque econométrico, y que se inspiran en la ley de gravitación universal de Newton. En el ámbito de la economía, estos modelos fueron inicialmente empleados por Reilly (1931) para analizar los factores que atraen el comercio minorista a ciudades más grandes. Más adelante, en los años 60 del siglo XX, estas modelizaciones se extendieron al contexto del comercio internacional (Tinbergen 1962; Pöyhönen, 1963). En este ámbito, los modelos de gravedad indican que los flujos comerciales entre países dependen directamente de sus tamaños, donde el tamaño de un país se puede medir mediante diferentes variables proxi ${ }^{1}$, como son su población o su riqueza, medida a través del PIB, y dependen inversamente de la distancia entre ellos.

Recientemente, se ha comenzado a utilizar este tipo de modelización para el análisis del flujo de turistas entre países, al entender, por un lado, que su valor está relacionado con la importancia de la riqueza de los países y la distancia entre los mismos, y por otro, que la demanda de turistas repercute directamente sobre la economía y riqueza de los países. Entre estos trabajos, cabe citar por ejemplo los de Khadaroo y Seetanah (2008) Fourie y SantanaGallego (2011), Fourie y Rosselló (2015) y Alawin y Abu-Lila (2016).

Estos estudios pueden modelizar el flujo turístico en una doble dirección (intercambio de turistas entre dos países), o bien, en dirección única, al considerar un país o región como foco atractivo. En este último caso, se modeliza la demanda de turistas hacia una zona desde otros países del entorno o más alejados.

Partiendo de estos estudios previos, y bajo el enfoque de dirección única, este trabajo tiene por objetivo modelizar la demanda de turismo que se genera en Andalucía (región del sur de España) desde los países europeos. Dos factores fundamentales, riqueza de un país (como medida de la masa del mismo) y distancia (desde dicho país a Andalucía), son determinantes en el flujo de turistas. Usamos esos dos factores con objeto de ser fiel a la modelización clásica inspirada en Newton. La medición del peso de cada uno de ellos para el caso de Andalucía, con respecto a los países europeos, es el objetivo de esta investigación. Para ello, se utiliza un modelo de gravedad en el que se analiza el flujo de turistas europeos que se alojaron en establecimientos hoteleros de Andalucía en 2017. Flores y Barroso (2012) muestran que, para un periodo de crecimiento económico, los valores de la elasticidad-renta se aproximan a la unidad, y añaden que "se puede concluir que el turismo internacional es una

1. En econometría, variable proxi es aquella que intenta representar a una variable no observada y, con la que suponemos existe una fuerte relación. 
actividad económica procíclica, con una gran sensibilidad a los ciclos económicos". Entonces, como 2017 es un año de expansión, de crecimiento económico, llevamos a cabo una estimación del mismo modelo para el caso del año 2011, con peor situación económica, y con objeto de comparar las estimaciones de parámetros y medir los cambios que se producen al pasar de un año a otro. Por tanto, la importancia de nuestro análisis radica en corroborar, de manera econométrica, la significatividad de las variables riqueza y distancia en la demanda turística de Andalucía, demanda medida a través de la ocupación hotelera, y la implicación fundamental de esta investigación es la medición tangible de esa efectividad y, por tanto, medición del posible cambio en la demanda ante cambios de las variables explicativas.

Centrar el estudio en Andalucía resulta de especial interés por dos motivos fundamentales. Por un lado, Andalucía es la región española que atrajo mayor número de turistas, tanto nacionales como internacionales, en $2016^{2}$, siendo España uno de los principales receptores de turismo en el mundo ${ }^{3}$. De este modo, el resultado del análisis puede servir para comprender mejor el efecto de eso dos factores en el movimiento internacional de turistas en Europa. Por otro lado, el peso del sector turístico sobre su actividad económica resulta de especial relevancia para la región, por lo que su análisis puede redundar en el desarrollo de políticas económicas relevantes para su economía.

Además, establecer como foco de estudio las llegadas de turistas desde Europa resulta también de especial interés por dos razones importantes para nosotros. En primer lugar, centrar el estudio solo en estos países elimina posibles distorsiones del modelo debido a los controles de acceso de población a España. Dada la fácil movilidad de personas entre países europeos, este posible efecto distorsionador queda eliminado. En segundo lugar, centrar el estudio en los países de Europa resulta significativo, ya que más del $80 \%$ de las pernoctaciones en hoteles por turistas extranjeros tuvo como procedencia países de Europa (INE, 2018). Resulta entonces que, centrarse en estos países, puede ayudar a pensar sobre la aplicación de medidas de política económica por parte de las autoridades autonómicas de la Junta de Andalucía.

A partir de este punto, este trabajo se divide en seis partes: Primero se pone de manifiesto la importancia del turismo en Andalucía. Tras ello se realiza una revisión de la literatura, y en las dos siguientes secciones se detalla la metodología y se describen los datos utilizados, respectivamente. En la siguiente se muestran los resultados y, finalmente, en la última presentamos nuestras conclusiones derivadas.

2. En la publicación Turismo Español en Andalucía, publicación oficial de la Consejería de Turismo y Deporte de la Junta de Andalucía, www.juntadeandalucia.es/turismoydeporte/publicaciones, para el año 2016, sitúa a Andalucía en el puesto no1 en el ranking de Comunidades Autónomas, en el movimiento hotelero español, con un $21^{\prime} 1 \%$ del total de pernoctaciones. Según el INE, en sus estadísticas sobre Movimientos Turísticos en Fronteras, en el Número de Turistas según comunidad autónoma de destino principal, en 2016 Andalucía ocupó en 4ㅇ lugar en España, con casi 11 millones de visitantes, tras Cataluña, Canarias y Baleares.

3. Según la Organización Mundial de Turismo, en su informe Panorama OMT, Edición 2017, https://www.e-unwto. org/doi/pdf/10.18111/9789284419043, España ocupó el tercer puesto del mundo en cuanto al número de visitantes en 2016, empatado con el segundo, Estados Unidos, con 75'6 millones, y el segundo puesto en cuanto a ingresos por turismo internacional, con $58^{\prime} 5$ miles de millones de dólares. 


\section{EL TURISMO EN LA ECONOMÍA ANDALUZA}

España constituye una de las principales potencias receptoras de turistas internacionales, tan solo por detrás de Francia y disputando un segundo lugar con Estados Unidos, según datos de 2017 según Memoria Anual TURESPAÑA 2017². Las causas apuntadas para el buen desarrollo de esta actividad pueden apuntarse a su alta competitividad. De hecho, el Foro Económico Mundial valoró a España como la nación con mayor nivel de competitividad turística en 2015, medida por el llamado Travel \& Tourism Competitiviness Index (TTCI) (WEF, 2017). Junto a su alta competitividad, otros factores pueden estar también influyendo en sus buenos resultados, como las situaciones de inestabilidad, de conflictos o de guerras, en zonas con atractivo turístico competencia natural de España, que han tenido un efecto positivo sobre el turismo español (Perles-Ribes, et al, 2016).

Dentro de España, Andalucía, recibió en 2016 un total de 28,2 millones de turistas, representando el $13,6 \%$ de total de pernoctaciones españolas en establecimientos hoteleros. Esto le hace consagrarse como la región española con mayor número de turistas totales (SAETA, 2018). Asimismo, en ese mismo año, Andalucía recibió 10 '75 millones de turistas extranjeros (SAETA, 2018), convirtiéndose en la cuarta comunidad española que recibió mayor número de turistas internacionales, tan solo detrás de Cataluña, y los dos archipiélagos. Los principales destinos dentro de la región han sido la Costa del Sol y Sierra Nevada.

La situación de Andalucía, al sur de la Península Ibérica, hace que sea uno de los lugares más cálidos de Europa. Predomina en todo el territorio el clima mediterráneo, que aporta un gran número de horas de sol, lo cual, junto con sus playas, configura las condiciones para el desarrollo turístico de "sol y playa". A ese turismo de sol y playa se añade, y cada vez con más fuerza, el turismo cultural. Andalucía cuenta con una gran riqueza patrimonial e histórica, lo que la hace situarse como región con altos niveles de capital turístico asociado a la cultura. Asimismo, Andalucía cuenta con un alto nivel de recursos naturales, que le han permitido desarrollar ampliamente su turismo de naturaleza. Así, en conjunto, la región cuenta con elevados índices de capital turístico asociado a la cultura, a la naturaleza y al tradicional de sol y playa (Sánchez-Braza, 2016).

La llegada de turistas y el gasto realizado por ellos, determinó que, en 2016 (según Balance de Año Turístico en Andalucía 2016, Junta de Andalucía, Consejería de Turismo y Deporte) $)^{5}$ los ingresos por turismo de la región fueran de 19,2 miles de millones de euros, lo que representa el $12,5 \%$ del PIB. Además, si a esto le añadimos los ingresos obtenidos por el excursionismo, la participación asciende hasta el 13,9\%. Por otra parte, el total de ocupados en actividades turísticas en Andalucía en 2017, según las mismas fuentes, ha sido 396.142, con un total de asalariados de 316.171 que, respecto al total de ocupados en todos los sectores en Andalucía, 2.971.613, nos muestra la importancia del sector turístico para la economía andaluza (un 13 '3 \%).

Cabe destacar también la tendencia alcista del turismo en la región. Durante 2016 y tras el repunte de 2015, se ha observado un gran dinamismo, influenciado más intensamente

4. https://www.tourspain.es/es-es/Conozcanos/MemoriasAnuales/MEMORIA\%202017\%20FINAL.pdf

5. http://www.juntadeandalucia.es/turismoydeporte/publicaciones/estadisticas/bata 2016.pdf 
por el turismo extranjero, registrándose un crecimiento real del 7,7\%, superior en 2,5 puntos porcentuales al registrado en 2015. En 2017, el número de turistas extranjeros que se alojaron en servicios hoteleros, según encuesta de ocupación hotelera del INE (2018), ha sido de 8.869.012 personas, con un crecimiento de un 7 ' $8 \%$ con respecto a 2016 .

\section{REVISIÓN DE LA LITERATURA}

El modelo de gravedad utilizado en las modelizaciones econométricas, se inspira en la famosa ley de gravitación universal de Newton:

$$
F=G \frac{m \cdot m^{\prime}}{d^{2}},
$$

donde $G$ es la constante de gravitación universal, $m$ y $m^{\prime}$ son las masas de los cuerpos que se atraen, $d$ es la distancia a la que se encuentran ambos cuerpos, y $F$ es la fuerza con la que ambos cuerpos se atraen.

La Ley de Newton, ampliamente utilizada por diversas ciencias, fue también bien recibida por la ciencia económica. La Ley de Gravitación Minorista de Reilly (1931), desarrollada en el ámbito de la economía urbana, en concreto en los factores que atraen el comercio minorista a ciudades más grandes, animó encarecidamente a otros autores a utilizar el mismo concepto de gravedad para otras áreas de investigación del campo de la economía. Así, en los años 60 del siglo XX se desarrollaron de forma independiente por Tinbergen (1962) y Pöyhönen (1963), las primeras aplicaciones de los modelos gravitacionales al comercio internacional para modelizar el flujo del comercio entre países. Para ello, utilizaron como variable proxy de las "masas" de los países, o bien el PIB de los mismos o bien el tamaño de población, y como distancia la propia distancia geográfica, convirtiéndose esta última como proxy del coste del transporte. En general, se puede argumentar que existe una motivación económica más fuerte para que el comercio tenga lugar entre dos países o regiones vecinas (al menos, debido a que los costos inherentes de transporte son menores). Este argumento, también conocido como disuasión por distancia, implica que los flujos comerciales entre dos áreas están inversamente relacionados con la distancia entre ellos. El amplio avance de estos modelos en el área de análisis económico de flujos comerciales está en el hecho de que tienen una base intuitiva muy fuerte, convirtiéndose, por tanto, en instrumentos simples e intuitivos para el análisis económico empírico. Así, durante mucho tiempo, la relevancia empírica de los modelos gravitacionales (ver, por ejemplo, Isard (1956) y Tinbergen (1962)) ha superado su base teórica.

No obstante, cabe decir que también se ha desarrollado una corriente que trata de realizar una fundamentación teórica de estos modelos. Encontramos, en los años 80 del siglo XX, algunos autores como Anderson (1979) y Bergstrand $(1985,1989)$, que han hecho fuertes contribuciones teóricas. Lo mismo sucedió con Anas (1983), quien demostró que los modelos de gravedad, como los de tipo logit, pueden derivarse de la maximización de una utilidad aleatoria. Más recientemente, Brakman et al. (2001), por ejemplo, afirma que el modelo gravitatorio fue más ampliamente aceptado por la teoría del comercio internacional, precisamente porque su ecuación básica puede derivarse de la nueva teoría del comercio. Fujita y Thisse (2002, p. 220), a propósito de la teoría de la formación de centros urbanos bajo competencia imperfecta, mencionan que la información incompleta de los consumidores es una fuerza 
aglutinadora ya que "la utilidad esperada de visitar un grupo de empresas aumenta con su tamaño, que es una reminiscencia del principio de gravedad".

Desde el punto de vista empírico, aunque como afirma Matías (2004), la ecuación de gravedad se utilizó fundamentalmente para explicar por qué los flujos comerciales eran más grandes entre los países vecinos (es decir, aquellos con límites terrestres), también se ha utilizado posteriormente para otros fines en la ciencia económica. Entre las diversas aplicaciones posibles de estos modelos, se debe enfatizar el estudio de los cambios ocurridos en estructuras de separación espacial (como peajes, puentes y nuevas carreteras), los efectos de la redistribución espacial de las actividades urbanas (por ejemplo, nuevas áreas residenciales, áreas industriales, supermercados, escuelas u hospitales), el desarrollo de modelos multirregionales de salida y de entrada, o el desgaste del espacio y los factores de atracción/rechazo presentes en diferentes lugares, solo por mencionar algunos. Ejemplos de variedad de esas aplicaciones citadas podemos observarlos en Aitken, N. D. (1973), Anas, A. (1983), Bergstrand, J. (1989), Brakman et al. (2001), Fujita, M. y Thisse, J. F. (2002) y Isard, W. (1956).

Este último aspecto es de suma importancia en el estudio del mercado turístico ya que el factor atracción es conocido como una de las variables fundamentales de la función de demanda turística. Así, Yang, Lin y Han (2010) analizan el papel de los Lugares de Patrimonio Mundial como factor de atracción para los turistas internacionales que viajan a China.

La prosperidad económica del período de la posguerra y el rápido desarrollo de los transportes, especialmente por vía aérea, ha hecho que el factor "distancia" aparezca como una variable importante en los nuevos enfoques sobre estudios de oportunidades de mercado, y en concreto del mercado del turismo. En este sentido, los flujos de turismo tienen un tratamiento muy similar al comercio de mercancías, donde la demanda estaría relacionada con el lugar de origen y la oferta con el destino. Como se trata de una industria basada en un bien negociable, cuya venta está intrínsecamente dirigida a mercados externos, y con el acto de consumo en función del desplazamiento del consumidor al mercado, es importante evaluar en qué medida la variable distancia es explicativa de los flujos turísticos hacia un determinado lugar.

Esto justifica la idoneidad de usar modelos de gravedad para analizar los determinantes de este sector donde, tanto las variables socioeconómicas como la distancia, juegan un importante papel. Dichos modelos, además, permiten cierta flexibilidad de manejo según la orientación del estudio. A pesar de esta importancia, el empleo de modelos de gravedad aplicados al turismo no se produce hasta el siglo XXI (Morley et al., 2014). Entre estos estudios, Khadaroo y Seetanah (2008) emplean un modelo de gravedad para evaluar la importancia de las infraestructuras del transporte en la determinación del atractivo turístico de los destinos. Keum (2010) explora la validez de la ecuación de la gravedad para explicar los flujos turísticos. Fourie y Santana-Gallego (2011) usan el modelo de gravedad para cuantificar los efectos de la celebración de grandes eventos deportivos sobre la llegada de turistas. Fourie y Rosselló (2015) usan este tipo de modelos para investigar el peso de la afiliación religiosa del país de destino en el flujo de viajeros. Alawin y Abu-Lila (2016) investigan el efecto de la incertidumbre sobre el turismo internacional de Jordania. Kaplan y Aktas (2016) utiliza este modelo para analizar la demanda turística en Turquía, desde una perspectiva bidireccional. En general, estos 
estudios pueden considerarse una forma de modelizar la función de demanda turística agregada basada en la teoría del consumidor (Morley et al., 2014).

En el caso de España, cabe decir que hasta dónde llega nuestro conocimiento, tan solo el estudio de Martí y Puertas (2017) analiza la demanda de turistas bajo el prisma de los modelos de gravedad. En el estudio se analiza los determinantes de los flujos de turistas domésticos. No obstante, no existe de momento ningún estudio que se centre en el flujo de turistas extranjeros.

El presente estudio amplia la evidencia empírica previa analizando el flujo de turistas extranjeros de 19 países europeos hacia Andalucía a través del uso de un modelo de gravedad de demanda, para el cual no existe evidencia empírica previa. Este estudio además presenta una novedad importante respecto a los estudios previos, pues determina el valor distancia, no solo por la distancia geográfica o real (e línea recta) sino también por la distancia medida en términos de acceso por carretera, lo que mejora las estimaciones realizadas.

\section{METOdOLOGÍA}

El modelo de la gravedad (en su forma estocástica), aplicado a los flujos comerciales, puede expresarse con la siguiente representación:

$$
T_{i j}=c \cdot P I B_{i}^{\beta_{1}} \cdot P I B_{j}^{\beta_{2}} \cdot d_{i j}^{\beta_{3}} \cdot \varepsilon_{i j},
$$

donde $T_{i j}$ es el flujo comercial entre los países $i$ y $j, P I B_{i}$ y $P I B_{j}$ representan el Producto Interior Bruto de los países $i$ y $j$ (usándola como variables proxis de las "masas" en la fórmula newtoniana), $d_{i j}$ es la distancia física entre ambos países y $\varepsilon_{i j}$ es un término de perturbación aleatoria que sigue un modelo log-normal. Los parámetros $\beta_{1}, \beta_{2}$ y $\beta_{3}$ representan las elasticidades del flujo comercial con respecto a las tres variables introducidas. Se espera, para ser coherente con el modelo de gravedad, que las dos primeras sean positivas y la tercera negativa. Aparece una constante, $c$, con objeto de dar libertad a la ecuación a la hora de establecer la relación entre el primer y segundo miembro. Sin dicha constante, la igualdad se hace más rígida. En el caso de la propuesta inicial de Newton, dicha $c$ se convirtió en la conocida como Constante de Gravitación Universal.

A partir de ese modelo, puede definirse un modelo gravitacional para estudiar los flujos de turismo europeo hacia Andalucía, desde la perspectiva de la demanda. En este caso, la demanda de turistas de países europeos puede expresarse como una función de su riqueza y de la distancia que los separa de Andalucía. De esta forma, el modelo adopta la siguiente expresión, en su forma estocástica:

$$
T_{i A}=c \cdot P I B_{i}^{\beta_{1}} \cdot d_{i A}^{\beta_{2}} \cdot \varepsilon_{i},
$$

donde $T_{i A}$ es el flujo de turistas desde el país europeo $i$ hacia Andalucía. En este caso, el flujo es medido por el número de turistas del país i que se alojaron en instalaciones hoteleras andaluzas durante el año 2017. La masa del país $i$ es aproximada mediante el PIB de dicho país, mientras $d_{i A}$ es la distancia desde el país $i$ a Andalucía, expresada en kilómetros. Su cálculo se detalla en la sección 5 de datos. $\varepsilon_{i}$ es el término de perturbación aleatoria. 
La ecuación anterior puede ser linealizada tomando logaritmo neperiano:

$$
\ln T_{i A}=\beta_{0}+\beta_{1} \cdot \ln P I B_{i}+\beta_{2} \cdot \ln d_{i A}+u_{i}, \quad i=1, \ldots, n,
$$

donde $u_{i}=\ln \varepsilon_{i}$ es el término de perturbación aleatoria del modelo lineal, con distribución normal de media cero y varianza constante para todos los valores de $i$; y $\beta_{0}=\ln c$ es la constante del modelo. Esta ecuación puede ser estimada por medio de Mínimos Cuadrados Ordinarios (MCO), eficiente para el caso de datos transversales que no presenten problemas de heteroscedasticidad.

En caso de que sea posible asumir la hipótesis de que $\beta_{1}=1$, el modelo (1) queda simplificado del siguiente modo:

$$
\ln T_{i A}=\beta_{0}+\ln P I B_{i}+\beta_{2} \cdot \ln d_{i A}+u_{i},
$$

por tanto,

$$
\ln T_{i A}-\ln P I B_{i}=\beta_{0}+\beta_{2} \cdot \ln d_{i A}+u_{i} .
$$

es decir

$$
\ln \left(\frac{T_{i A}}{P I B_{i}}\right)=\beta_{0}+\beta_{2} \cdot \ln d_{i A}+u_{i},
$$

quedando entonces como variable a modelizar el número de turistas alojados en establecimiento hoteleros relativizados por el PIB de sus correspondientes países, una especie de densidad o ratio de turistas alojados por millón de $€$ de PIB.

Si el logaritmo neperiano de la ratio entre turistas y PIB se expresa como $T_{i A}^{r}$, la ecuación (1) se expresa finalmente como sigue:

$$
\ln T_{i A}^{r}=\beta_{0}+\beta_{2} \cdot \ln d_{i A}+u_{i}
$$

es decir, el logaritmo de la ratio de turistas alojados en establecimiento hoteleros por millón de euros del PIB se modeliza mediante una única variable explicativa, el logaritmo de la distancia. De este modo, si se relativizan los flujos turísticos procedentes de un país respecto a su propio PIB, la distancia se convierte en la variable clave.

En este sentido, cabe esperar que los flujos turísticos dirigidos hacia un destino continental, como es el caso de Andalucía, exhiban una correlación negativa con respecto a la distancia desde el país de origen, ya que los costos de transporte son menores para distancias más cortas. Téngase en cuenta que, si el país de destino tiene límites terrestres con el país de origen (como es el caso de Portugal), incluso es posible usar el transporte en automóvil como alternativa al avión. Además, la alternativa del automóvil se vuelve más viable en un espacio económico unificado, con factores y bienes que se mueven libremente, como es el caso actual de la Unión Europea. Naturalmente, aunque la distancia es solo uno entre los diversos factores que determinan los flujos de turismo, la misma impacta en el precio final de los productos turísticos debido a la agravación de los costos de transporte, lo que no deja dudas sobre su influencia negativa en esos flujos. 
Por tanto, si de la modelización resulta que la distancia es un factor relevante para la demanda turística y si podemos cuantificar su relevancia, la cuestión de promover el destino turístico andaluz puede basarse en una intervención por parte de la administración pública en este sector, a través de políticas públicas que haga que el efecto contrario de la distancia disminuya, optimizando de esta forma el potencial de este sector para crear riqueza.

\section{DATOS}

Los datos sobre flujo de turistas en Andalucía se refieren a número de turistas totales procedentes de 19 países europeos que se alojaron en establecimientos hoteleros de la región durante el año 2017. Estos datos proceden de SAETA (2018) y son construidos a partir de la Encuesta de Ocupación Hotelera del INE. Los correspondientes al PIB de los 19 países que forman parte de nuestro estudio proceden de Eurostat (2018). Están valorados en millones de euros del año 2016, último año para el que están disponibles datos completos. A partir de los datos anteriores se puede calcular el número de turistas alojados en establecimientos hoteleros por millón de $€$ del PIB, lo que muestra una medida de densidad de turistas. La construcción de esta última variable ha sido inducida por la aparición del modelo (2) que es consecuencia del modelo (1) para el caso en que se diese, en el mismo, la relación 1 a 1 entre las variables "logaritmo de turistas" y "logaritmo de PIB", o sea, cuando se acepta que la pendiente de esa última variable es 1 en ese modelo (1), lo cual ocurrirá, como veremos más abajo, con los datos usados para este año. Obviamente, la variable resultante, densidad de turistas por PIB, puede resultar a priori artificiosa, pero puede ser una buena medida de atracción del turismo hacia Andalucía desde los diferentes países, si estos son uniformados por el valor de la riqueza.

La variable "distancia" desde cada país a Andalucía, ha sido construida como media ponderada de dos distancias: distancia en línea recta entre la capital del país y la capital andaluza, Sevilla, medida en kilómetros (representando así la distancia recorrida por los turistas que se desplazaron en avión) y la distancia por carretera entre las dos ciudades mencionadas, medida también en kilómetros (representando la distancia recorrida por el resto de turistas). La ponderación de la media se calcula en base al porcentaje de turistas alojados en hoteles en 2017 que se desplazaron en avión frente al total. La Tabla 1 muestra los datos correspondientes a esta variable: 
Tabla 1. Construcción de los datos muestrales empleados en la variable distancia

\begin{tabular}{|c|c|c|c|c|c|}
\hline País & $\begin{array}{l}\text { Distancia en } \\
\text { línea recta, en } \\
\text { kms. }\end{array}$ & $\begin{array}{c}\text { \% de turistas } \\
\text { que llegan en } \\
\text { avión }\end{array}$ & $\begin{array}{l}\text { Distancia por } \\
\text { carretera, en } \\
\text { kms. }\end{array}$ & $\begin{array}{l}\% \text { turistas que } \\
\text { llegan por otros } \\
\text { medios }\end{array}$ & $\begin{array}{c}\text { Distancia } \\
\text { ponderada, en } \\
\text { kms. }\end{array}$ \\
\hline Alemania & 2257 & 82,7 & 2835 & 17,3 & 2356,994 \\
\hline Austria & 2172 & 76,7 & 2806 & 23,3 & 2319,722 \\
\hline Bélgica & 1705 & 71,9 & 2038 & 28,1 & 1798,573 \\
\hline Dinamarca & 2463 & 73,0 & 2947 & 27,0 & 2593,68 \\
\hline Finlandia & 3338 & 75,0 & 4267 & 25,0 & 3570,25 \\
\hline Francia & 1441 & 59,0 & 1734 & 41,0 & 1561,13 \\
\hline Grecia & 2604 & 83,0 & 4077 & 17,0 & 2854,41 \\
\hline Holanda & 1869 & 71,9 & 2234 & 28,1 & 1971,565 \\
\hline Irlanda & 1775 & 78,0 & 2748 & 22,0 & 1989,06 \\
\hline Italia & 1656 & 48,0 & 2339 & 52,0 & 2011,16 \\
\hline Luxemburgo & 1689 & 70,0 & 2153 & 30,0 & 1828,2 \\
\hline Noruega & 2775 & 77,0 & 3424 & 23,0 & 2924,27 \\
\hline Polonia & 2668 & 81,0 & 3375 & 19,0 & 2802,33 \\
\hline Portugal & 313 & 18,0 & 393 & 82,0 & 378,6 \\
\hline Reino Unido & 1638 & 78,0 & 2188 & 22,0 & 1759 \\
\hline Rep. Checa & 2151 & 65,0 & 2695 & 35,0 & 2341,4 \\
\hline Rusia & 3822 & 83,0 & 4584 & 17,0 & 3951,54 \\
\hline Suecia & 2983 & 75,0 & 3550 & 25,0 & 3124,75 \\
\hline \multirow[t]{2}{*}{ Suiza } & 1532 & 53,0 & 1927 & 47,0 & 1717,65 \\
\hline & $\%$ medio & 69,4 & $\%$ medio & 30,6 & \\
\hline
\end{tabular}

Fuente: Construcción propia a partir de datos de http://www.distanciasentreciudades.com y de datos de SAETA (2018).

La Tabla 2 presenta los datos muestrales correspondientes a las variables utilizados en la estimación de las ecuaciones [1] y [2]. 
Tabla 2. Datos

\begin{tabular}{|c|c|c|c|c|}
\hline País & $\begin{array}{l}\text { Número de viajeros } \\
\text { europeos en } \\
\text { establecimientos } \\
\text { hoteleros. } \\
\text { Año } 2017\end{array}$ & $\begin{array}{c}\text { PIB } 2016 \\
\text { En millones de } €\end{array}$ & $\begin{array}{c}\text { Distancia } \\
\text { ponderada, en kms. }\end{array}$ & $\begin{array}{c}\text { Densidad de } \\
\text { turistas alojados } \\
\text { por millón de } € \text { de } \\
\text { PIB }\end{array}$ \\
\hline Alemania & 925312 & 3144050.0 & 2356.994 & 0.2943 \\
\hline Austria & 69526 & 353296.9 & 2319.722 & 0.1968 \\
\hline Bélgica & 273741 & 423048.4 & 1798.573 & 0.6471 \\
\hline Dinamarca & 127073 & 277489.1 & 2593.680 & 0.4579 \\
\hline Finlandia & 74358 & 215773.0 & 3570.250 & 0.3446 \\
\hline Francia & 844895 & 2228857.0 & 1561.130 & 0.3791 \\
\hline Grecia & 17461 & 174199.3 & 2854.410 & 0.1002 \\
\hline Holanda & 417812 & 702641.0 & 1971.565 & 0.5946 \\
\hline Irlanda & 196150 & 275567.1 & 1989.060 & 0.7118 \\
\hline Italia & 500365 & 1680522.8 & 2011.160 & 0.2977 \\
\hline Luxemburgo & 14520 & 53004.8 & 1828.200 & 0.2739 \\
\hline Noruega & 96727 & 335503.8 & 2924.270 & 0.2883 \\
\hline Polonia & 137454 & 425980.2 & 2802.330 & 0.3227 \\
\hline Portugal & 243905 & 185179.5 & 378.600 & 1.3171 \\
\hline Reino Unido & 1549105 & 2395801.0 & 1759.000 & 0.6466 \\
\hline Rep. Checa & 41227 & 176564.3 & 2341.400 & 0.2335 \\
\hline Rusia & 92212 & 1159546.0 & 3951.540 & 0.0795 \\
\hline Suecia & 178698 & 465186.2 & 3124.750 & 0.3841 \\
\hline Suiza & 152799 & 604455.9 & 1717.650 & 0.2528 \\
\hline Total & 5.953 .340 & & & \\
\hline
\end{tabular}

Fuente: Construcción propia a partir de datos de datos de SAETA (2018) y EUROSTAT (2018).

Cabe destacar el valor que toma la variable de densidad, mostrado en la última columna, para el caso de Portugal, 1.3171, muy superior con diferencia a todos los demás. Asimismo, destaca el inferior valor de la distancia (columna 3), pues es el único país extranjero con frontera con Andalucía. De este modo, como Portugal es el único país extranjero con frontera común con Andalucía, es muy posible que la mayoría de los visitantes procedentes de ese país (82\% según encuesta de ocupación hotelera) usen el vehículo propio como alternativa al avión, haciendo más barato el coste de aproximación.

En este sentido, cabe señalar la relación inversa entre las variables de densidad y distancia, siendo la correlación entre ambas variables $-0.7012(p=0.008)$. Asimismo, la Figura 1 muestra el diagrama de dispersión densidad (turistas alojados por millón de $€$ de PIB) versus distancia en kilómetros. Se observa una relación claramente decreciente ente ambas. 
Figura 1. Diagrama de dispersión densidad de turistas alojados versus distancia

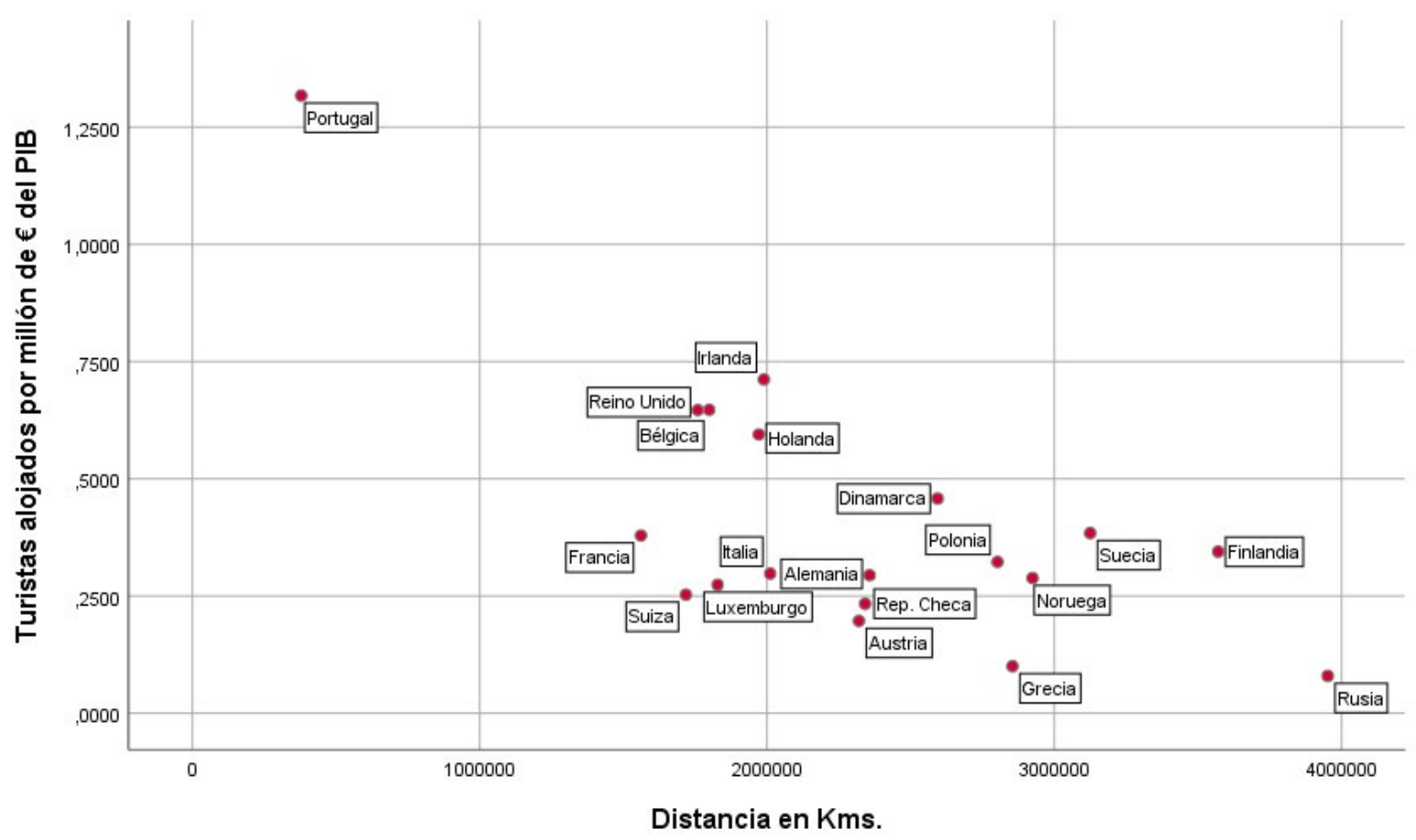

Fuente: Construcción propia a partir de datos de http://www.distanciasentreciudades.com, de datos de SAETA (2018) y EUROESTAT (2018).

\section{RESULTADOS}

La Tabla 3 muestra los resultados de estimar la ecuación [1] por MCO.

Tabla 3. Estimación por MCO del modelo (1) para datos 2016/2017

\begin{tabular}{|l|c|c|c|c|c|}
\hline & Coeficiente & Error estándar & Estadístico $t$ & valor $p$ & \\
\hline Constante & 5.40093 & 2.35730 & 2.291 & 0.0359 & $* *$ \\
\hline Ln (PIB) & 1.03243 & 0.116210 & 8.884 & $<0.0001$ & $* * *$ \\
\hline Ln (Distancia) & -0.90219 & 0.251065 & -3.593 & 0.0024 & $* * *$ \\
\hline R-cuadrado & 0.845172 &
\end{tabular}

** Significatividad al 5\%,***Significatividad al $1 \%$

Elaboración propia usando como soporte de cálculo el software libre GRETL.

Usamos el PIB del país y no el PIB per cápita porque el objetivo de nuestra modelización es usar una variable proxi del tamaño o masa del país. Así, se da el caso de un país como Luxemburgo, el de mayor renta per cápita, que aporta un número reducido de turistas debido, lógicamente, a que el país es pequeño. La filosofía implícita en los modelos de gravedad es la de usar la masa total del país, y no la masa per cápita.

Los resultados mostrados en la Tabla 3 indican que el PIB de los países visitantes tiene un impacto positivo en los flujos de turismo hacia Andalucía, una vez que la existencia de un alto nivel de poder adquisitivo en el país de origen es un determinante básico a la existencia 
de la demanda turística en sí. Entonces, el tamaño relativo de los países (medido por su PIB) afecta directamente al número de turistas que visitan la región. Asimismo, los resultados indican que la distancia entre las dos localizaciones afecta negativamente al flujo turístico. Los resultados indican asimismo que la elasticidad del flujo de turistas de un país con respecto a la riqueza del mismo país (1.03243) supera, en valor absoluto, a la elasticidad del flujo de turistas con respecto a la distancia (0.90219).

Con el fin de mostrar la robustez de los resultados, se han llevado a cabo una serie de análisis de los mismos. En primer lugar, la Figura 2 muestra la relación de los valores observados y ajustados de acuerdo con la estimación de [1] entre los turistas alojados de cada uno de los países considerados (en logaritmo neperiano, In) y el PIB de dichos países (en logaritmo neperiano, In). La proximidad entre valores observados y ajustados nos informa de la bondad del ajuste. El nivel de dispersión de la variable a modelizar (número de viajeros europeos en establecimientos hoteleros andaluces en 2017) ha permitido un buen ajuste del modelo, dado el $R^{2}$ resultante $(0.845172)$.

Figura 2. Diagrama de dispersión para valores observados y ajustados del $\ln$ (turistas) versus $\ln (\mathrm{PIB})$

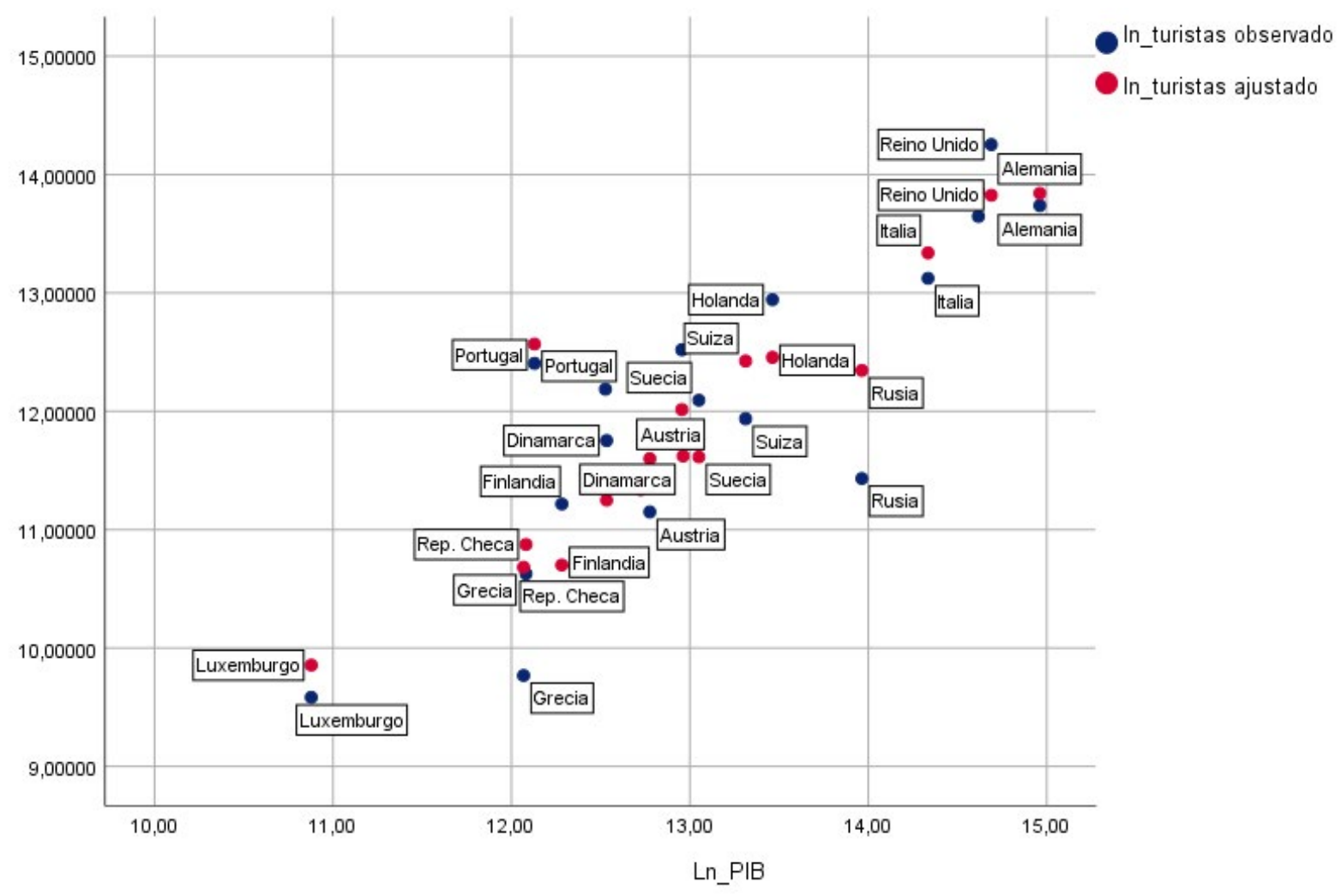

Fuente: Elaboración propia a partir de datos de datos de SAETA (2018) y EUROESTAT (2018).

En segundo lugar, se ha analizado la homocedasticidad de los residuos para comprobar si el modelo por MCO es eficiente. Así, se han llevado a cabo los contrastes de heteroscedasticidad de White, de Breusch-Pagan, y el de Breusch-Pagan, con la variante robusta de Koenker 
(Wooldridge, 2006) ${ }^{6}$. Los resultados de dichos contrastes aparecen en la Tabla 4. Todos los resultados indican la no existencia de problemas de heteroscedasticidad, por lo que la hipótesis de varianza común entre las perturbaciones aleatorias, exigida para la estimación eficiente del método MCO se sostiene.

Tabla 4. Contrastes de heteroscedasticidad en el modelo (1)

\begin{tabular}{|l|c|c|}
\hline \multicolumn{1}{|c|}{ Contraste } & Estadístico del contraste & Valor $p$ \\
\hline White & $\mathrm{TR}^{2}=4.940727$ & $0.423157>0.05$ \\
\hline Breusch-Pagan & $\mathrm{LM}=1.723296$ & $0.422465>0.05$ \\
\hline $\begin{array}{l}\text { Breusch-Pagan (versión } \\
\text { robusta de Koenker) }\end{array}$ & $\mathrm{LM}=3.140057$ & $0.208039>0.05$ \\
\hline
\end{tabular}

Elaboración propia usando como soporte de cálculo el software libre GRETL.

Asimismo, se ha analizado la posible colinealidad de las variables del modelo, indicando que el modelo estimado no presenta problemas de ese tipo, entre las dos variables explicativas. La correlación entre el PIB de los países de la muestra y la distancia de los mismos a Andalucía es $-0.1112(p=0.6502)$ y la correlación entre sus respectivos logaritmos es $0.079(p=0.7477)$. El factor de inflación de la varianza ha sido 1.006 para ambas variables explicativas (muy por debajo del valor 10 usado como frontera para la posible presencia de colinelaidad) y el número de condición toma el valor 42.38 (muy por debajo de 75, usado como valor mínimo para la posible presencia de este problema si se intenta detectar mediante el número de condición).

Por último, los diferentes contrastes realizados no permiten rechazar la hipótesis nula de normalidad para los residuos. La Tabla 5 muestra los resultados de 4 contrastes de normalidad distintos. Observamos que todos ellos nos llevan a no rechazar dicha hipótesis.

Tabla 5. Contrastes de normalidad de los residuos en la estimación del modelo (1)

\begin{tabular}{|l|c|c|}
\hline \multicolumn{1}{|c|}{ Contraste } & Estadístico del contraste & Valor $p$ \\
\hline Doornik-Hansen & 1.25688 & $0.533423>0.05$ \\
\hline W de Shapiro-Wilk & 0.920259 & $0.114423>0.05$ \\
\hline Lilliefors & 0.176312 & $\sim=0.12>0.05$ \\
\hline Jarque-Bera & 1.03343 & $0.596477>0.05$ \\
\hline
\end{tabular}

Elaboración propia usando como soporte de cálculo el software libre GRETL

De este modo, la estimación realizada por MCO mantiene las hipótesis exigidas a las perturbaciones aleatorias, por lo que podemos considerar eficientes las estimaciones de los parámetros, y en particular las de las dos elasticidades.

Dado que el valor de la elasticidad para el PIB es 1.03243, cabe plantearse la posibilidad de contrastar la hipótesis de que dicha elasticidad sea igual a 1. El estadístico F asociado

6. En las páginas 295-299 Wooldrige recomienda el uso de estas pruebas diagnósticas para detectar la presencia de heteroscedasticidad en un modelo estimado. 
al contraste de esta restricción lineal, $\beta_{1}=1$, ha tomado el valor $0.0778908(p=0.783752)$, lo que nos permite el sostenimiento de dicha hipótesis. De este modo es posible estimar la ecuación (2), referida a la ratio de turistas alojados en establecimiento hoteleros por millón de euros del PIB.

La Tabla 6 muestra los resultados de estimar la ecuación [2] por MCO.

Tabla 6. Estimación por MCO del modelo (2)

\begin{tabular}{|l|c|c|c|c|c|}
\hline & Coeficiente & $\begin{array}{c}\text { Error } \\
\text { estándar }\end{array}$ & Estadístico t & valor $p$ & \\
\hline Constante & 5.78237 & 1.86785 & 3.096 & 0.0066 & $* * *$ \\
\hline Ln (Distancia ponderada) & -0.896660 & 0.243397 & -3.684 & 0.0018 & $* * *$ \\
\hline R-cuadrado & 0.443925 & $* * *$ Significatividad al 1\% \\
\cline { 2 - 4 } &
\end{tabular}

Puede observarse en la Tabla 6 que la elasticidad entre el flujo de turistas relativizado por el PIB y la distancia, prácticamente no ha variado con respecto a la elasticidad obtenida respecto de la variable distancia de la ecuación [1]. Así, el valor de la elasticidad en el primer modelo fue -0.90 y en el segundo -0.89 . O sea, el efecto contrario de la distancia sobre el flujo de viajeros es prácticamente el mismo tanto si se considera dicho flujo en términos absolutos (y se modeliza acompañado del PIB) como si se hace en términos relativos. Dicha elasticidad nos informa que, imaginando de manera ficticia, un alejamiento geográfico de un país de procedencia de turistas de tal manera que suponga un incremento de distancia de un $10 \%$, implicaría una disminución de turistas alojados de un $9 \%$. Dicho de otra forma, hay una relación contraria entre turista alojados y distancia de 10 a 9. La estimación de este modelo proporciona una medida empírica del peso que tiene la distancia en la demanda turística andaluza.

Si observamos el modelo (2) como caso particular del modelo (1), el resultado obtenido está en línea con el trabajo desarrollado por Brakman et al. (2001) (pp.12-15), que sugiere efectuar una corrección sobre los flujos comerciales en el caso de la demanda que genera una masa económica más grande de los países de mayor tamaño. Esto corresponde, por lo tanto, a un caso particular en nuestro modelo (1) donde la elasticidad número de turistas/riqueza es igual a uno.

Como ya se comentó, realizamos una estimación del modelo de gravedad inicial para datos del año 2011. Los datos son extraídos de las mismas fuentes, y los resultados de la estimación aparece en la Tabla 8. Los datos usados son los que aparecen en la Tabla 7. 
Tabla 7. Datos para la estimación MCO para año 2011

\begin{tabular}{|c|c|c|c|}
\hline País & $\begin{array}{l}\text { Número de viajeros } \\
\text { europeos en } \\
\text { establecimientos } \\
\text { hoteleros. } \\
\text { Año } 2017\end{array}$ & $\begin{array}{c}\text { PIB } 2016 \\
\text { En millones de } €\end{array}$ & $\begin{array}{c}\text { Distancia } \\
\text { ponderada, en } \\
\text { kms. }\end{array}$ \\
\hline Alemania & 768053 & 2703120 & 2466,63 \\
\hline Austria & 53291 & 310129 & 2420,83 \\
\hline Bélgica & 183481 & 379106 & 1822,86 \\
\hline Dinamarca & 66700 & 247880 & 2630,79 \\
\hline Finlandia & 40120 & 196869 & 3678,61 \\
\hline Francia & 658611 & 2058369 & 1544,25 \\
\hline Grecia & 998569 & 1894900 & 1826,29 \\
\hline Holanda & 14074 & 207029 & 3122,05 \\
\hline Irlanda & 293980 & 650359 & 1996,18 \\
\hline Italia & 106406 & 171140 & 2114,49 \\
\hline Luxemburgo & 454496 & 1637463 & 1897,61 \\
\hline Noruega & 10230 & 43165 & 1825,29 \\
\hline Polonia & 60541 & 358734 & 3040,24 \\
\hline Portugal & 97252 & 380242 & 2921,46 \\
\hline Reino Unido & 193623 & 176167 & 365,65 \\
\hline Rep. Checa & 31478 & 164041 & 2335,36 \\
\hline Rusia & 75518 & 1474456 & 4104,42 \\
\hline Suecia & 67544 & 405440 & 3195,82 \\
\hline Suiza & 90546 & 504021 & 1697,17 \\
\hline Total & 4264512 & & \\
\hline
\end{tabular}

Fuente: Construcción propia a partir de datos de datos de SAETA (2012) y EUROSTAT (2012).

Tabla 8. Estimación por MCO del modelo (1) para datos de 2011

\begin{tabular}{|l|c|c|c|c|l|}
\hline & Coeficiente & Error estándar & Estadístico $t$ & valor $p$ & \\
\hline Constante & 6.25501 & 2.246950 & 2.784 & 0.0133 & $* *$ \\
\hline Ln (PIB) & 1.02700 & 0.113707 & 9.032 & $<0.0001$ & $* * *$ \\
\hline Ln (Distancia) & -1.04044 & 0.240363 & -4.329 & 0.0005 & $* * *$ \\
\hline R-cuadrado & 0.853730 & \multicolumn{4}{ll}{} \\
\cline { 1 - 5 } & &
\end{tabular}

** Significatividad al 5\%, ***Significatividad al $1 \%$

Elaboración propia usando como soporte de cálculo el software libre GRETL.

La Tabla 9 recoge la comparación de coeficientes estimados en ambas regresiones. Observamos la poca diferencia existente, sobre todo en la elasticidad riqueza/número de turistas. O sea, en el caso de Andalucía, y para turistas europeos alojados en establecimientos hoteleros, dicha elasticidad estuvo alrededor de 1, tanto en un año de crisis, 2011, como en un año de expansión, 2017. La elasticidad distancia/alojamiento de turistas era más negativa 
en 2011. O sea, la distancia era más disuasoria para la llegada de turistas a Andalucía en 2011 que en 2017.

Tabla 9. Coeficientes estimados por MCO del modelo (1) para datos de 2011 y 2016/2017

\begin{tabular}{|l|c|c|}
\hline & Datos 2011 & Datos 2016/2017 \\
\hline Constante & 6.25501 & 5.40093 \\
\hline Ln (PIB) & 1.02700 & 1.03243 \\
\hline Ln (Distancia) & -1.04044 & -0.90219 \\
\hline
\end{tabular}

Elaboración propia usando como soporte de cálculo el software libre GRETL.

\section{CONCLUSIÓN}

Teniendo en cuenta que el turismo se va asumiendo cada vez con más fuerza como una actividad económica organizada, surge la necesidad de establecer un desarrollo acorde de su marco teórico. En nuestro caso lo hemos buscado a través de modelizaciones específicas relacionadas inicialmente con los flujos comerciales. La metodología econométrica nos ha sido útil para concluir relaciones significativas entre las variables usadas.

Utilizando un modelo de gravedad simple, hemos demostrado que la riqueza de un país que emite turistas a Andalucía tiene una relación 1:1 con respecto al número de turistas procedentes del mismo y que se aloja en establecimientos hoteleros, si hablamos en términos porcentuales, o sea, un incremento de un 15 de la riqueza genera un incremento de igual porcentaje en el número de turistas alojados en Andalucía. Dicha relación se ha dado tanto en un año de expansión económica, 2016 como en un año de peor situación, 2011. Y que la distancia en 2016/2017 tuvo una elasticidad negativa de 0.9 lo que supuso una reducción con respecto a 2011, que fue de 1.04. Cabe pensar que, el abaratamiento de los costes de transporte, por el incremento de líneas de bajo coste, haya sido un factor influyente en la bajada de dicha elasticidad. También podemos afirmar que las elasticidades de ambos factores, riqueza y distancia, son casi iguales y de signo contrario (podemos hablar de una relación de 10:9).

Una vez que esa distancia se acepta como un factor relevante para la demanda turística, se deben considerar las implicaciones de este resultado en el tema de la promoción de la marca Andalucía como destino turístico. Es decir, cuando se considera junto con las características específicas del bien turístico, la importancia empírica de la distancia en este contexto, nuestro resultado sugiere que puede haber algún tipo de intervención por parte de las autoridades de regulación del sector turístico que permitan optimizar el enorme potencial de este sector para crear riqueza económica, con diferentes enfoques según que se trate de países cercanos o países alejados de Andalucía. Dichas intervenciones han de ir en la dirección de abaratamiento de costes de transportes, que es una forma de reducir la distancia. 


\section{REFERENCIAS BIBLIOGRÁFICAS}

Aitken, N. D. (1973). The Effect of the EEC and EFTA on European Trade: A Temporal CrossSection Analysis. American Economic Review, 63, 881-892.

Alawin, M., Abu-Lila, Z. (2016). Uncertainty and Gravity Model for international Tourism Demand in Jordan: Evidence from Panel-GARCH model. Applied Econometrics and International Development, 16 (1), 131-146.

Anas, A. (1983). Discrete choice theory, information theory, and the multinomial logit and gravity models. Transportation Research, 17, 13-23.

Anderson, J. (1979). A Theoretical Foundation for the Gravity Equation. American Economic Review, 69, 106-116.

Arezki, R., Cherif, R., Piotrowski, J. (2009). Tourism Specialization and Economic Development: Evidence from the UNESCO world Heritage List. IMF Working Paper, Wp/09/176.

Bergstrand, J. (1985). The Gravity Equation in International Trade: Some Microeconomic Foundations and Empirical Evidence. Review of Economics and Statistics, 67, (3), 474-481.

Bergstrand, J. (1989). The Generalized Gravity Equation, Monopolistic Competition, and the Factor-Proportions Theory in International Trade. Review of Economics and Statistics, 71, (1), 143-153.

Brakman, S., Garretsen, H., Van Marrewijk, C. (2001). An Introduction to Geographical Economics. Cambridge University Press.

Flores Ruiz, D., Barroso González, M. O. (2012). La demanda turística internacional. Medio siglo de evolución. Revista de Economía Mundial, 32, 127-149.

Fourie, J., Rosselló, J. (2015). Religion, Religious Diversity and Tourism. Kyklos, 68 (1), 51-64.

Fourie, J., Santana-Gallego, M. (2011). The impact of mega-sport events on tourist arrivals. Tourism Management, 32, 1364-1370.

Freytag, A., Vietze, C. (2010). Can Nature Promote Development? The Role of Sustainable Tourism for Economic Growth. Jena Economic Research Paper 08/2010, 1-32.

Fujita, M., Thisse, J. F. (2002). Economics of Agglomeration-Cities, Industrial Location, and Regional Growth. Cambridge University Press.

Gerakis, A. S. (1965). Effect of Exchange-rate Devaluations and Revaluations on Receipts from Tourism. International Monetary Fund Staff Papers, 12, 365-384.

Gray, H. P. (1966). The Demand for International Travel by United states and Canada. International Economic Review, 7, 83-92.

Guthrie, H. W. (1961). Demand for Tourists' Goods and Services in a World Market. Papers and Proceedings of the Regional Science Association. 7, 159-175.

Isard, W. (1956). Location and the Space Economy. John Wiley, New York.

Kaplan, F., and Aktas, A. R. (2016). The Turkey Tourism Demand: A Gravity Model. The Empirical Economics Letters, 15(3), 265-272.

Keum, K. (2010). Tourism flows and trade theory: a panel data analysis with the gravity model. The Annals of Regional Science, 44(3), 541-557

Khadaroo, J., Seetanah, B. (2008). The role of transport infrastructure in international tourism development: A gravity model approach. Tourism Management, 29, 831-840.

Matias, Á. (2004). Gravity and the tourism trade: the case for Portugal. Sustainable Tourism, F. D. Pineda, C. A. Brebbia \& M. Mugica (Editors), 47-64. 
Morley, C., Rosselló, J., and Santana-Gallego, M. (2014). Gravity models for tourism demand: theory and use. Annals of Tourism Research, 48, 1-10.

Perles-Ribes, J. F., Ramón-Rodríguez, A. B., Moreno-Izquierdo, L., Torregrosa Martí, M. T. (2016). Winners and losers in the Arab uprisings: a Mediterranean tourism perspective. Current Issues in Tourism. dx.doi.org/10.1080/13683500.2016.1225697

Pöyhönen, P. (1963). A Tentative Model for the Volume of Trade Between Countries. Weltwirtschaftliches Archiv, 90 (1), 93-99

Reilly, W. (1931). The Law of Retail Gravitation. New York, Pillsbury.

Santeramo, F. G., Morelli, M. (2016). Modelling tourism flows through gravity models: a quantile regression approach. Current Issues in Tourism, 19 (11), 1077-1083.

Song, H., Li, G. (2008). Tourism Demand Modeling and Forecasting. A Review of Resent Research. Tourism Management, 29, 203-220.

Tinbergen, J. (1962). Shaping the World Economy: Suggestions for an International Economic Policy. New York, Twentieth Century Fund.

Woodridge, J. M (2006). Introducción a la econometría. Un enfoque moderno. 2a edición. International Thomson Editores Spain Paraninfo, S. A. Madrid.

Yang, C. H., Lin, H. L., Han, C. C. (2010). Analysis of international tourist arrivals in China: The role of World Heritage Sites. Tourism Management, 31, 827-837. 